\title{
Comparação do estado nutricional em pacientes com insuficiência renal crônica com e sem hiperparatireoidismo secundário
}

\author{
Comparison of nutritional status in patients with chronic renal \\ failure with and without secondary hyperparathyroidism
}

Aryell David Proença', Carolina Lacorte Galina', Luisa Godoi Lopes', Monike Santana Gobbo', Victor Ribeiro Miamoto', Yuri Gam Faria', João Victor Marques Guedes', Márcia Christina Caetano Romano', Fernanda Cecília Dias Chula $^{2}$, Yoshimi Jose Ávila Watanabe ${ }^{2}$, Alba Otoni

'Universidade Federal de São João del-Rei, Campus CCO/Divinópolis/MG

${ }^{2}$ Hospital São João de Deus /Divinópolis/Minas Gerais - Brazil

E-mail para contato: Aryell David Proença - aryellproenca@hotmail.com

\section{Resumo}

Objetivo: Comparar, por meio de instrumento específico (Malnutrition Inflammation Score - MIS) e recordatório alimentar, o perfil nutricional de pacientes com Insuficiência Renal Crônica (IRC) em hemodiálise com e sem Hiperparatireoidismo Secundário (HPT2). Métodos: estudo transversal com 204 pacientes com IRC em hemodiálise com e sem HPT2. O estado nutricional foi avaliado por meio do instrumento MIS e a ingesta alimentar por recordatório 24h, sendo dados deste último analisados pelo software Avanutri®. Resultados: Do total de pacientes, 136 tinham HPT2 e 68 não tinham, sendo a maioria do sexo masculino, etilistas e sedentários. Em relação ao MIS, o número de pacientes com pontuação > 6 (indicando risco nutricional) foi de 48,5\% para aqueles sem HPT2 e 49,3\% para os com HPT2, porém, não houve diferença estatística entre esses grupos ( $p>0,05)$. Com relação ao consumo de micronutrientes, identificou-se que as pessoas com IRC e HPT2 consumiram em maior quantidade todos os micronutrientes, com exceção a vitamina B12. No que diz respeito aqueles micronutrientes que podem intervir diretamente no HPT2, a vitamina D e o fósforo foram significativamente mais consumidos pelo grupo com IRC e HPT2. Conclusão: Não houve diferença estatística no estado nutricional de pacientes com IRC em hemodiálise com e sem HPT2. Os pacientes com IRC e HPT2 consumiram em maior quantidade todos os micronutrientes, em especial de vitamina $D$ e fósforo, porém mantiveram os níveis séricos adequados podendo indicar, o atendimento de recomendações específicas para tratamento do HPT2.

Palavras-chave: Insuficiência Renal Crônica. Doença renal crônica. Diálise Renal. Estado Nutricional. Hiperparatireoidismo Secundário.

\begin{abstract}
Objective: To compare by means of a specific instrument (Malnutrition Inflammation Score - MIS) and dietary recall, the nutritional profile of patients with chronic renal failure (CRF) on hemodialysis with and without Secondary Hyperparathyroidism (HPT2). Methods: cross-sectional study with 204 patients with CRF on hemodialysis with and without HPT2. Nutritional status was assessed
\end{abstract}


by means of the MIS instrument and food intake by 24-hour recall. Data from the latter was analyzed using Avanutri® software. Results: Of the total patients, 136 had HPT2 and 68 did not, most of them being male, alcoholic and sedentary in both groups. Regarding MIS, validated instrument to assess nutritional risk, the number of patients with a score $>6$ (indicating nutritional risk) was $48.5 \%$ for those without HPT2 and $49.3 \%$ for those with HPT2, however, there was no statistical difference between the groups ( $p>0.05$ ). Regarding the consumption of micronutrients, it was found that people with CRF and HPT2 consumed in greater quantities all micronutrients, except for vitamin B12. With regard to micronutrients that can directly intervene in HPT2, vitamin D and phosphorus were significantly more consumed by the group with CRF and HPT2.Conclusion: There was no statistical difference in the nutritional status of patients with CRF on hemodialysis with and without HPT2. Patients with CRF and HPT2 consumed a greater amount of all micronutrients, especially vitamin $D$ and phosphorus, but maintained adequate serum levels, which may indicate the fulfillment of specific recommendations for the treatment of HPT2.

Keywords: Chronic Renal Insufficiency. Chronic Kidney Disease. Renal Dialysis. Nutritional Status. Secondary Hyperparathyroidism.

\section{INTRODUÇÃO}

As doenças do rim e do trato urinário são responsáveis por aproximadamente 850 milhões de mortes anuais em todo mundo e a incidência da Insuficiência Renal Crônica (IRC) aumenta em torno de $8 \%$ ao ano ${ }^{1}$. Mesmo diante da clara definição do diagnóstico da IRC, ainda é exorbitante o número de pessoas que chegam à atenção secundária especializada sem qualquer abordagem anterior na atenção primária com vistas à preservação das funções renais e mesmo controle da evolução para Insuficiência renal crônica quando a IRC já se encontra instalada ${ }^{2,3}$. No momento em que os pacientes com doença renal evoluem para a IRC/doença crônica terminal (DRCT), a abordagem com as terapias renais substitutivas (TRS) são as alternativas para manutenção da vida e equilíbrio hemodinâmico desses pacientes. E apesar do avanço e da alta tecnologia utilizada nas TRS, diante do complexo contexto que envolve a doença renal, muitos distúrbios metabólicos não são totalmente controlados e predispõem às complicações clínicas ${ }^{4}$.

Entre estas complicações encontra-se o Distúrbio Mineral e Ósseo (DMO) que se configura em manifestações que envolvem anormalidades nos níveis de cálcio, fósforo, paratormônio (PTH), ou do metabolismo da vitamina $\mathrm{D}$, anormalidades na remodelação óssea, mineralização, crescimento linear e calcificações extraesqueléticas. 
O DMO pode iniciar-se em estágios precoces da IRC e considera-se que a retenção do fósforo, causada pela redução da Taxa de Filtração Glomerular (TFG), seja o fator primário do desenvolvimento do hiperparatireoidismo secundário (HPT2), pois induz a hipocalcemia, a redução na produção de calcitriol e aumento da secreção do $\mathrm{PTH}^{5,6}$. Em recente e importante estudo, os pesquisadores identificaram que em 11 centros com 1134 pacientes em diálise, a prevalência de pacientes fora do alvo para PTH foi de 50,5\%7. Devido ao quadro instalado de desordem no equilíbrio de fósforo, cálcio e hormônios reguladores, a dieta adequada de níveis calóricos e fósforo exerce influência essencial no estado nutricional do paciente com IRC, em especial, naqueles já em TRS.

Durante a hemodiálise (HD), as escórias nitrogenadas são removidas para o alcance dos níveis séricos compatíveis com o bem-estar do paciente, pois por mais adequadas que sejam, as TRS não conseguem substituir totalmente as funções renais a ponto de reduzir os níveis séricos das escórias para níveis considerados fisiológicos.

Além disso, perdas de aminoácidos podem ocorrer de forma significativa para a solução dialítica a ser desprezada, e se não forem repostas adequadamente a fim de suprir tais demandas, poderá levar a um quadro de desnutrição energética proteica (DEP). Essa desnutrição acontece quando há uma insuficiente oferta de calorias ou de proteínas ou quando essas são retiradas pelo tratamento dialítico e não repostas. Associado a essa perda involuntária, está a proposta de restrição de fósforo para controle de níveis séricos, que se não for adequadamente ajustada à ingestão proteica, poderá comprometer ainda mais o estado nutricional do paciente, e implicar em um impacto negativo no prognóstico da IRC ${ }^{8}$.

Diante desse cenário, considera-se grande o desafio para a equipe multiprofissional assistencial, o controle do estado nutricional do paciente com IRC em TRS quando se tem como foco manter a oferta adequada de proteína, sem aumentar os níveis de fósforo. Questiona-se se os pacientes com HPT2 que têm orientação nutricional de restrição fosfórica teriam um estado nutricional mais comprometido no que diz respeito aos desafios de ajustes da nutrição energético proteica.

Neste sentido, o objetivo desse estudo foi comparar por meio de instrumento específico e recordatório alimentar, o perfil nutricional de pacientes com IRC em hemodiálise com e sem HPT2. 
Aryell David Proença, Carolina Lacorte Galina, Luísa Godoi Lopes, Monike Santana Gobbo, Victor Ribeiro Miamoto, Yuri Gam Faria, João Victor Marques Guedes, Márcia Christina Caetano Romano, Fernanda Cecília Dias Chula, Yoshimi Jose Ávila Watanabe, Alba Otoni | Comparação do estado nutricional em pacientes com insuficiência renal crônica com e sem hiperparatireoidismo secundário

\section{MÉTODO}

\section{Pacientes e delineamento do estudo}

Trata-se de um estudo transversal realizado em uma unidade de nefrologia de um hospital de grande porte situado na região ampliada de saúde do Centro-Oeste de Minas Gerais no período de março de 2017 a julho de 2019.

Foram incluídos todos os pacientes adultos de ambos os sexos que estavam em hemodiálise há pelo menos seis meses com diagnóstico de HPT2. Para efeito de comparação foi incluído no estudo outro grupo de pacientes adultos de ambos os sexos em hemodiálise há pelo menos seis meses, sem diagnóstico de HPT2. O tempo mínimo de HD de seis meses, após início da TRS, em ambos os grupos condiz com o intervalo necessário estimado para o desenvolvimento de complicações, como o DMO e o HPT2. Os critérios de exclusão foram pacientes com câncer terminal e com diagnóstico de desnutrição por outras causas distintas.

Para realização da coleta de dados os pesquisadores foram treinados acerca da adequada abordagem do estado nutricional. Após treinamento, os dados foram coletados e o questionário Malnutrition Inflammation Score (MIS) aplicado por: enfermeiros, graduandos em medicina, médicos e nutricionista. Inicialmente se analisou os prontuários, e os pacientes que preenchiam os critérios de inclusão foram convidados a participar do estudo. Os dados foram coletados de fonte primária com entrevista e exame físico dos dados antropométricos durante as sessões de HD e as informações faltantes foram complementadas a partir de consultas em fonte secundária (prontuário). Foram coletadas variáveis sociodemográficas (sexo, idade, estado civil, escolaridade e cor da pele), relativas ao comportamento de saúde (alcoolismo e tabagismo registrados com o auto relato de sim ou não, sedentarismo, considerando como prática de atividade qualquer tipo de atividade física), características clínicas (Índice de Massa Corporal (IMC), Pressão Arterial Sistólica (PAS), Pressão Arterial Diastólica (PAD), doença de base da DRC, outras comorbidades associadas e tempo em HD), uso de medicamentos, dados laboratoriais coletados dos prontuários (níveis séricos de creatinina, cálcio, fósforo, PTH, proteínas totais e fracionadas), além das informações nutricionais descritas no MIS e no recordatório alimentar, detalhados a seguir.

Considerou-se hiperparatireoidismo secundário quando a dosagem sérica de paratormônio intacto era maior ou igual a $300 \mathrm{pg} / \mathrm{mL}^{9,10}$. 
O perfil nutricional foi analisado por meio do instrumento específico para avaliação do risco nutricional em pacientes renais: o Malnutrition Inflammation Score (MIS) ${ }^{11}$, no qual por meio de 10 componentes avaliados e pontuados, (informações da história clínica, do exame físico e de condição laboratorial) define-se a presença ou ausência de risco nutricional. $\mathrm{O}$ ponto de corte definido para classificação da presença ou ausência do risco nutricional foi a soma da pontuação igual a seis, ou seja, variável dicotômica $\geq$ a 6 indica risco nutricional, < 6 indica que não há risco nutricional ${ }^{11-13}$. O escore final a ser avaliado para a caracterização desse risco considera níveis numéricos extremos que variam entre o normal (0) ao severamente desnutrido (30). Salienta-se que quanto maior a numeração na direção do número 30, pior a condição nutricional do paciente.

Ainda foi aplicado o Recordatório Alimentar de 24 horas, que trata de um inquérito alimentar e consiste em definir e quantificar todos os alimentos e bebidas ingeridas no dia anterior à entrevista. A partir dos alimentos ingeridos foram quantificadas calorias, valores de macro e micronutrientes com auxílio do software Avanutri versão online ${ }^{\circledR}$.

\section{Análise estatística}

Os dados coletados foram inseridos com dupla entrada do banco EpiData ${ }^{\circledR} 3.1$ e posteriormente foram exportados e analisadas pelo Statistical Package for Social Sciences ${ }^{\circledR}$, versão 21.0 (SPSS, Inc., Chicago, IL, EUA). Foi realizada análise descritiva a fim de caracterizar a população do estudo. Para as variáveis categóricas foram empregadas tabelas de distribuição de frequência. Para as variáveis contínuas foram adotadas medidas de tendência central e variabilidade sendo a mediana e percentis utilizados como medida de variabilidade quando as variáveis contínuas analisadas não apresentaram distribuição normal e média e desvio padrão quando apresentaram distribuição normal (Shapiro-Wilk). Para comparação entre os grupos com e sem HPT2 foram utilizados os testes qui-quadrado $\left(\mathrm{X}^{2}\right)$ de Pearson e exato de Fischer. A associação entre o risco nutricional (MIS) e a presença de HPT2 foi estimada por meio da regressão logística. Foram estimados os OddsRatios (OR), brutos e ajustados e os seus respectivos intervalos de confiança de 95\% (IC 95\%). Primeiramente estimou-se o OR bruto da associação entre o risco nutricional e a presença de HPT2 (Modelo 0), a seguir, o OR bruto foi ajustado por sexo, idade, estado civil e escolaridade (Modelo 1). Posteriormente ajustou-se o OR por atividade 
física, tabagismo, etilismo e seguir orientações alimentares (Modelo 2). Por fim o modelo 3 foi ajustado por uso de antidepressivos, benzodiazepínicos, presença de distúrbios metabólicos, respiratórios, cardiovasculares, hepáticos, sintomas gastrointestinais e capacidade funcional. As variáveis de ajuste ou eram sabidamente associadas ao estado nutricional ou tiveram de $\mathrm{p}<0,20$ na análise univariada. A pesquisa foi aprovada pelo Comitê de Ética em Pesquisa Envolvendo Seres Humanos do Campus Centro Oeste Dona Lindu-UFSJ sob o CAAE 61654216.6.0000.5545 parecer $\mathrm{n}^{\mathrm{o}} 1.896 .709$.

\section{RESULTADOS}

De março a outubro de 2017, todos os pacientes em HD que preenchiam os critérios de inclusão, além daqueles sorteados para comporem o grupo de comparação (sem HPT2) foram incluídos no estudo totalizando 204 pacientes. Desses, 136 (66,6\%) tinham HPT2 e 68 não eram acometidos por essa condição de saúde. As características sociodemográficas e as variáveis de comportamento de saúde dos participantes estão demonstradas na Tabela 1. Dentre os pacientes que não possuíam HPT2, 18 (26,4\%) tiveram como doença de base da IRC a hipertensão arterial sistêmica (HAS), $18(26,4 \%)$ o diabetes mellitus (DM) e o restante foi acometido por outras doenças de base menos frequentes como, por exemplo, rins policísticos, que estavam presentes em quatro pacientes (5,9\%). Já entre aqueles que possuíam HPT2, a maioria também teve a HAS com causadora da IRC, com 49 pacientes (36\%) e o DM com 34 (25\%). Os demais tiveram outras doenças de base menos frequente (Dados não incluídos em tabela).

Em relação às variáveis clínicas do estudo, os resultados apontam que os pacientes com HPT2 estavam em terapia renal substitutiva há mais tempo que aqueles sem HPT2, sendo suas medianas, respectivamente, 65,5 meses e 42 meses. Quanto aos valores da Pressão Arterial Sistólica (PAS), a mediana em pacientes com HPT2 encontrava-se maior: $140 \mathrm{mmHg}$ contra $135 \mathrm{mmHg}$ dos pacientes sem HPT2, enquanto a diastólica manteve-se igual entre os dois grupos $80 \mathrm{mmHg}$. Já entre os resultados obtidos pelo recordatório alimentar, destaca-se: a mediana do Valor Calórico Total (VCT) de 897,83 kcal no grupo sem HPT2 e de 879,82 no grupo com HPT2, ( $>>0,05)$. Entretanto, observou-se uma maior ingesta calórica relativa ao peso corporal no grupo com HPT2 $(25,07 \mathrm{kcal} / \mathrm{kg}$ de peso corpóreo) em relação ao grupo sem HPT2 $(17,83 \mathrm{kcal} / \mathrm{kg}$ de peso) com $\mathrm{p}<0,05$. Os dados obtidos do 
recordatório alimentar demonstraram diferenças significativas na ingestão de proteína absoluta (45,33 g sem HPT2 e 56,45 g com HPT2; p<0,05), porém não na ingesta relativa $(0,72 \mathrm{~g} / \mathrm{kg}$ nos sem HPT2 e $0,86 \mathrm{~g} / \mathrm{kg}$ nos com HPT2; p>0,05), nem dos demais macronutrientes (carboidratos e lipídeos). Também houve diferença significativa na ingesta de fósforo (494,095 mg sem HPT2 e 560,245 mg com HPT2; $\mathrm{p}<0,05)$. Algumas informações complementares quanto ao estado nutricional da população do estudo estão presentes na tabela 2 e os dados obtidos recordatório alimentar de 24 horas estão apresentados na tabela 3.

Tabela 1 - Dados sociodemográficos e as variáveis de comportamento de saúde de pacientes em hemodiálise no período de Março/2017 a Outubro/2017, em Divinópolis-MG/Brasil

\begin{tabular}{|c|c|c|}
\hline \multirow[t]{2}{*}{ Variáveis Sociodemográficas } & \multicolumn{2}{|c|}{ Hiperparatireoidismo Secundário (HPT2) } \\
\hline & Sem HPT2 (n=68) & Com HPT2 $(n=136)$ \\
\hline $\begin{array}{l}\text { Sexo } \\
\text { Masculino }\end{array}$ & $40(58,8 \%)$ & $89(65,4 \%)$ \\
\hline $\begin{array}{l}\text { Idade } \\
\text { Média }\end{array}$ & 59,26 & 54,85 \\
\hline $\begin{array}{l}\text { Cor da Pele } \\
\text { Branca } \\
\text { Parda } \\
\text { Negra } \\
\text { Amarela }\end{array}$ & $\begin{array}{c}27(39,7 \%) \\
22(32,4 \%) \\
17(25 \%) \\
1(1,5 \%)\end{array}$ & $\begin{array}{c}53(39,0 \%) \\
49(36,0 \%) \\
34(25 \%)\end{array}$ \\
\hline $\begin{array}{l}\text { Estado Civil } \\
\text { Casado/União Estável } \\
\text { Divorciado } \\
\text { Solteiro } \\
\text { Viúvo }\end{array}$ & $\begin{array}{c}38(55,9 \%) \\
10(14,7 \%) \\
14(20,6 \%) \\
6(8,8 \%)\end{array}$ & $\begin{array}{c}67(49.3 \%) \\
16(11,8 \%) \\
34(25,0 \%) \\
18(13,2 \%)\end{array}$ \\
\hline $\begin{array}{l}\text { Escolaridade } \\
\text { Analfabeto } \\
\text { Ensino Fundamental } \\
\text { Ensino Médio } \\
\text { Ensino Superior }\end{array}$ & $\begin{array}{c}8(11,8 \%) \\
36(52,9 \%) \\
16(23,5 \%) \\
8(11,8 \%)\end{array}$ & $\begin{array}{c}4(2,9 \%) \\
99(71,3 \%) \\
29(21,3 \%) \\
6(4,4 \%)\end{array}$ \\
\hline $\begin{array}{l}\text { Pratica atividade física } \\
\text { Sim } \\
\text { Não }\end{array}$ & $\begin{array}{l}17(25 \%) \\
51(75 \%)\end{array}$ & $\begin{array}{c}32(23,5 \%) \\
104(76,5 \%)\end{array}$ \\
\hline $\begin{array}{l}\text { Frequência semanal dos } \\
\text { praticantes } \\
1-2 \\
3 \text { ou mais }\end{array}$ & $\begin{array}{c}7(10,3 \%) \\
10(14,7 \%)\end{array}$ & $\begin{array}{c}11(8,1 \%) \\
21(15,4 \%)\end{array}$ \\
\hline $\begin{array}{l}\text { Fumantes } \\
\text { Nunca fumou } \\
\text { Fuma/ já fumou }\end{array}$ & $\begin{array}{l}34(50 \%) \\
34(50 \%)\end{array}$ & $\begin{array}{l}64(47,1 \%) \\
72(53,0 \%)\end{array}$ \\
\hline $\begin{array}{l}\text { Uso de Álcool } \\
\text { Nunca utilizou } \\
\text { Faz / fez uso }\end{array}$ & $\begin{array}{l}23(33,8 \%) \\
45(66,2 \%)\end{array}$ & $\begin{array}{c}32(23,5 \%) \\
104(76,4 \%)\end{array}$ \\
\hline
\end{tabular}


Aryell David Proença, Carolina Lacorte Galina, Luísa Godoi Lopes, Monike Santana Gobbo, Victor Ribeiro Miamoto, Yuri Gam Faria, João Victor Marques Guedes, Márcia Christina Caetano Romano, Fernanda Cecília Dias Chula, Yoshimi Jose Ávila Watanabe, Alba Otoni I Comparação do estado nutricional em pacientes com insuficiência renal crônica com e sem hiperparatireoidismo secundário

Tabela 2 - Dados complementares sobre o estado nutricional de pacientes em hemodiálise com Hiperparatireoidismo Secundário no período de Março/2017 a Outubro/2017, em Divinópolis-MG/Brasil. (n=204)

\begin{tabular}{l|c|c|c}
\hline \multirow{2}{*}{ Variáveis nutricionais } & \multicolumn{2}{|c|}{ Hiperparatireoidismo Secundário (HPT2) } & P-valor \\
\cline { 2 - 3 } & Sem HPT2 (n=68) & Com HPT2 (n=136) & \\
\hline Recebimento de informações & & & $0,70^{*}$ \\
nutricionais & $67(98,5 \%)$ & $134(98,5 \%)$ & \\
Sim & $1(1,5 \%)$ & $2(1,5 \%)$ & \\
Não & $48(70,6 \%)$ & $74(54,4 \%)$ & $0,01^{*}$ \\
Segue as recomendações & $20(29,4 \%)$ & $62(45,6 \%)$ & \\
Sim & & & \\
Não & $23,21(16,9-40,93)$ & $23,59(14,53-38,18)$ & $0,73^{*}$ \\
IMC & $7(10,44 \%)$ & $16(11,85 \%)$ & \\
Mediana & $36(52,23 \%)$ & $66(48,14 \%)$ & \\
Desnutrido & $16(23,88 \%)$ & $31(22,96 \%)$ & \\
Eutrófico & $9(13,43 \%)$ & $23(17,03 \%)$ & \\
Sobrepeso & & & $0,52^{*}$ \\
Obesidade & $35(51,50 \%)$ & $69(50,70 \%)$ & \\
Somatório MIS & $33(48,50 \%)$ & $67(49,30 \%)$ & \\
Menor ou igual a 6 & & & \\
Maior que 6 & & & \\
& & & \\
\hline
\end{tabular}

*Teste de $\mathrm{X}^{2}$

Tabela 3 - Ingestão dietética de pacientes em hemodiálise no período de Março/2017 a Outubro/2017, em DivinópolisMG/Brasil

\begin{tabular}{|c|c|c|c|}
\hline \multirow{2}{*}{$\begin{array}{l}\text { Ingestão Dietética dos } \\
\text { Pacientes } \\
\text { (Energia, macro e } \\
\text { micronutrientes) }\end{array}$} & \multicolumn{2}{|c|}{ Hiperparatireoidismo Secundário (HPT2) } & \multirow[t]{2}{*}{ P valor* } \\
\hline & $\begin{array}{c}\text { Sem HPT2 } \\
\text { Mediana (P25 - P75) e } \\
\text { média ( } \pm \text { DP) }\end{array}$ & $\begin{array}{c}\text { Com HPT2 } \\
\text { Mediana (P25 e P75) e média } \\
( \pm \text { DP) }\end{array}$ & \\
\hline VCT (Kcal) & $897,83(477,92-1381,53)$ & $879,82(25,46-1327,24)$ & 0,250 \\
\hline $\mathrm{Kcal} / \mathrm{Kg}$ de peso & $17,83(11,86-46,03)$ & $25,07(16,57-151,95)$ & 0,002 \\
\hline $\operatorname{Ptn}(\mathrm{g}) / \mathrm{Kg}$ de Peso & $0,72(0,44-1,11)$ & $0,86(0,58-1,24)$ & 0,099 \\
\hline Total Ptn (g) & $45,33(29,59-75,24)$ & $56,45(39,78-81,01)$ & 0,035 \\
\hline \% Ptn (kcal) & $18,66(13,41-22,56)$ & $16,87(14,13-22,00)$ & 0,729 \\
\hline Total Cho (g) & $153,28(111,37-196,24)$ & $169,09(125,50-238,17)$ & 0,034 \\
\hline$\%$ Cho (kcal) & $56,09( \pm 11,85)$ & $55,47( \pm 11,10)$ & 0,712 \\
\hline Total Lip (g) & $30,28(16,33-48,79)$ & $36,66(22,90-50,82)$ & 0,068 \\
\hline$\%$ Lip (kcal) & $25,6( \pm 10,77)$ & $26,14( \pm 9,55)$ & 0,757 \\
\hline Vitamina A (mg) & $144,28(27,23-480,85)$ & $216,74(104,53-490,11)$ & 0,047 \\
\hline Vitamina D (mcg) & $0,335(0,0575-0,800)$ & $0,57(0,180-1,087)$ & 0,044 \\
\hline Vitamina B1 (mg) & $0,665(0,380-1,520)$ & $0,875(0,577-1,677)$ & 0,021 \\
\hline Vitamina B2 (mg) & $0,5(0,23-0,84)$ & $0,58(0,37-0,91)$ & 0,079 \\
\hline Vitamina B5 (mg) & $1,11(0,632-1,787)$ & $1,6(0,912-2,757)$ & 0,002 \\
\hline Vitamina B6 (mg) & $0,56(0,29-0,98)$ & $0,585(0,362-1,052)$ & 0,345 \\
\hline Vitamina B12 (mg) & $1,27(0,19-3,08)$ & $0,79(0,26-2,68)$ & 0,587 \\
\hline Vitamina C (mg) & $5,03(0,95-28,93)$ & $8,5(1,56-34,25)$ & 0,137 \\
\hline Vitamina $\mathrm{E}$ (mg) & $3,5(1,92-6,34)$ & $4,745(2,520-10,762)$ & 0,019 \\
\hline Folato (mcg) & $27,31(14,74-56,06)$ & $36,94(18,47-82,81)$ & 0,105 \\
\hline Cálcio (mcg) & $158,05(98,05-243,33)$ & $170,04(113,21-332,80)$ & 0,116 \\
\hline Fósforo (mg) & $494,09(285,31-771,25)$ & $560,24(417,34-836,27)$ & 0,042 \\
\hline Magnésio (mg) & $97,27(64,89-172,74)$ & $122,68(84,62-173,98)$ & 0,050 \\
\hline Ferro (mg) & $8,145(4,27-12,27)$ & $8,845(6,887-13,752)$ & 0,028 \\
\hline
\end{tabular}


Aryell David Proença, Carolina Lacorte Galina, Luísa Godoi Lopes, Monike Santana Gobbo, Victor Ribeiro Miamoto, Yuri Gam Faria, João Victor Marques Guedes, Márcia Christina Caetano Romano, Fernanda Cecília Dias Chula, Yoshimi Jose Ávila Watanabe, Alba Otoni | Comparação do estado nutricional em pacientes com insuficiência renal crônica com e sem hiperparatireoidismo secundário

Continuação da tabela 3

\begin{tabular}{|c|c|c|c|}
\hline \multirow{2}{*}{$\begin{array}{l}\text { Ingestão Dietética dos } \\
\text { Pacientes } \\
\text { (Energia, macro e } \\
\text { micronutrientes) }\end{array}$} & \multicolumn{2}{|c|}{ Hiperparatireoidismo Secundário (HPT2) } & \multirow[t]{2}{*}{ P valor* } \\
\hline & $\begin{array}{c}\text { Sem HPT2 } \\
\text { Mediana (P25 - P75) e } \\
\text { média }( \pm D P)\end{array}$ & $\begin{array}{c}\text { Com HPT2 } \\
\text { Mediana (P25 e P75) e média } \\
( \pm \text { DP) }\end{array}$ & \\
\hline $\begin{array}{l}\text { Zinco }(\mathrm{mg}) \\
\text { Cobre }(\mathrm{mcg}) \\
\text { lodo }(\mathrm{mcg}) \\
\text { Selênio }(\mathrm{mcg}) \\
\text { Manganês }(\mathrm{mg}) \\
\text { Potássio }(\mathrm{mg}) \\
\text { Sódio }(\mathrm{mg}) \\
\end{array}$ & $\begin{array}{c}6,705(2,65-11,29) \\
0,49(0,31-0,87) \\
0,335(0,10-4,96) \\
26,015(8,95-40,90) \\
1,23(0,58-1,95) \\
909,42(563,59-1454,60) \\
851,49(514,87-1469,91) \\
\end{array}$ & $\begin{array}{c}6,83(4,37-10,47) \\
0,6(0,42-0,88) \\
0,785(0,12-10,64) \\
36,48(17,18-52,07) \\
1,57(0,89-2,10) \\
1101,86(711,5-1599,6) \\
1173,90(723,2-1948,9) \\
\end{array}$ & $\begin{array}{l}0,405 \\
0,052 \\
0,287 \\
0,020 \\
0,072 \\
0,070 \\
0,007 \\
\end{array}$ \\
\hline
\end{tabular}

* Teste de T Student Simples para comparação de médias ou Teste de Mann Whitney para comparação de medianas. (Kg: quilograma; g: grama; mg: miligrama; mcg: micrograma; Kcal: quilocalorias; Ptn: proteína; Cho: carboidrato; Lip: lipídeo;)

Os dados laboratoriais também foram analisados e pacientes com HPT2 obtiveram valores médios de creatinina maiores que o outro grupo, 9,65 e 8,42 mg/dL ( $p<0,05)$, respectivamente. Ambos os grupos apresentaram valores de fósforo sérico semelhantes: $4,20 \mathrm{mg} / \mathrm{dL}$ para os pacientes sem HPT2 e 4,70 $\mathrm{mg} / \mathrm{dL}$ para os com HPT2. A mediana de proteína total foi maior no grupo sem HPT2, 7,15 g/dL contra $7,12 \mathrm{~g} / \mathrm{dL}$ no outro grupo, com p>0,05. Em relação aos níveis séricos de paratormônio, o grupo com HPT2 apresentou valores maiores, como o esperado, mediana de 758,50 pg/ml comparada com 157,5 $\mathrm{pg} / \mathrm{ml}$ no grupo sem HPT2. A mediana dos valores de albumina foi a mesma em ambos os grupos, $4,20 \mathrm{~g} / \mathrm{dL}(\mathrm{p}>0,05)$.

Os resultados da análise multivariada de regressão logística e oddsratio ajustado correlacionando a presença de HPT2 e risco nutricional no período do estudo estão presentes na tabela 4, discriminando o OR bruto e associado a três diferentes modelos.

Tabela 4 - Análise multivariada Oddsratio (OR) bruto e ajustado para as associações entre o risco nutricional e a presença de HPT2 no período de Março/2017 a Outubro/2017, em Divinópolis-MG/Brasil

\begin{tabular}{l|c|c|c|c}
\hline \multirow{2}{*}{ Variável } & \multirow{2}{*}{ OR bruto } & \multicolumn{3}{|c}{ OR ajustado } \\
\cline { 2 - 5 } & & Modelo 1 & Modelo 2 & Modelo 3 \\
\cline { 2 - 5 } & $\mathbf{( I C ~ 9 5 \% )}$ & $\mathbf{( I C ~ 9 5 \% )}$ & $\mathbf{( I C} 95 \%)$ & (IC 95\%) \\
\hline Risco nutricional & & & & \\
Não & - & - & - & - \\
Sim & 1,02 & 1,12 & 1,05 & 0,87 \\
P-valor & $(0,57-1,84)$ & $(0,58-2,16)$ & $(0,53-2,06)$ & $(0,38-1,98)$ \\
\hline
\end{tabular}

Modelo 1: ajustado por sexo, idade, estado civil e escolaridade.

Modelo 2: modelo 1 + ajustado por atividade física, tabagismo, etilismo e seguir orientações alimentares.

Modelo 3: modelo 2 + ajustado para uso de antidepressivos, benzodiazepínicos, presença de distúrbios metabólicos, respiratórios, cardiovasculares, hepáticos, sintomas gastrointestinais e capacidade funcional.

\section{DISCUSSÃO}

A manutenção do estado nutricional adequado de pacientes com IRC e HPT2 é um desafio diário para toda equipe de saúde que assiste essa população, em especial, considerando a necessidade de ingestão restrita de fósforo e, de maneira simultânea, o equilíbrio da ingesta proteica. A utilização de evidências científicas acerca da abordagem nutricional ideal, seria uma sólida estratégia de referência para os 
profissionais que atuam na área da nefrologia. No entanto, a literatura com foco nutricional para essas pessoas é escassa e limitada, talvez pela dificuldade de quantificar e validar informações colhidas a respeito da dieta. Nesse sentido, considerando trabalhos semelhantes a este, nossos achados corroboram os resultados de Rezende et al. (2000)14 que não encontraram diferença do estado nutricional dos pacientes com HPT2 e os outros pacientes em HD. Esses autores entenderam que seus resultados poderiam ser justificados por uma subnotificação e/ou erros sistemáticos na estimativa de consumo alimentar dos pacientes. Por mais que informem um consumo próximo ao real, há um limite para quantificação de energia, macro e micronutrientes e, por isso, os estudos apresentam resultados que, embora mensuráveis e representativos do estado geral de nutrição, não conseguem apresentar um indicador que confirme se há ou não o seguimento correto da dieta recomendada.

Por outro lado, os resultados gerais desse estudo podem ser um indicativo da boa qualidade dos serviços prestados na unidade de saúde investigada. Como não houve diferença do risco nutricional entre os dois grupos, sugere-se que, possivelmente, os profissionais alcançaram os objetivos nutricionais que foram estabelecidos ao orientar a população a respeito da dieta a ser seguida ${ }^{15,16}$.

A presente investigação buscou também identificar diferenças entre o consumo alimentar de micronutrientes e observou que houve maior consumo de todos os micronutrientes pelos participantes com IRC e HPT2, com exceção da Vitamina B12. Ao contrário desses resultados, Campos et al. (2012) ${ }^{17}$ não identificaram diferenças de consumo alimentar(micronutrientes) entre pacientes com e sem HPT2. Os autores argumentaram que é necessário, nessa análise, considerar variáveis importantes na determinação do consumo de micronutrientes, como por exemplo, acesso a determinados tipos de alimentos, dificuldade de compreensão da orientação dada, entre outras. Afirmaram que essa é uma limitação em seu estudo que possivelmente pode ter influenciado nos resultados.

Cabe ressaltar que, sobre os determinantes do HPT2, a hipovitaminose D e a hiperfosfatemia têm papel de destaque ${ }^{10}$. De fato, as diretrizes brasileiras para prevenção e tratamento do HPT2 secundário na DRC, recomendam não somente dosar a vitamina $\mathrm{D}$, como promover orientação dietética para manutenção adequada dos níveis séricos deste micronutriente. Provavelmente, o atendimento a essas recomendações, prestadas de forma eficiente pelo serviço de saúde, justificam o 
Aryell David Proença, Carolina Lacorte Galina, Luísa Godoi Lopes, Monike Santana Gobbo, Victor Ribeiro Miamoto, Yuri Gam Faria, João Victor Marques Guedes, Márcia Christina Caetano Romano, Fernanda Cecília Dias Chula, Yoshimi Jose Ávila Watanabe, Alba Otoni | Comparação do estado nutricional em pacientes com insuficiência renal crônica com e sem hiperparatireoidismo secundário

maior consumo deste micronutriente pelos pacientes com HPT2 nesta investigação ${ }^{18}$.

Seguir de forma disciplinada a dieta prescrita é a ferramenta chave no controle do estado nutricional das pessoas com HPT2 à IRC. A respeito especificamente da ingestão de fósforo, recomenda-se de 800-1000 mg/dia para o paciente em hemodiálise, uma vez que valores acima destes podem contribuir para o desequilíbrio já existente da relação cálcio/fósforo séricos e, ainda, potencializar a fisiopatologia do $\mathrm{HPT}^{19}$.

No presente trabalho foi identificado que uma porcentagem menor dos pacientes com HPT2 afirmou seguir as recomendações nutricionais para restrição de fósforo quando comparados com os pacientes sem esse distúrbio. Mesmo assim, em ambos os grupos a ingestão desse micronutriente foi abaixo do recomendado pelas diretrizes do KDIGO $(2017)^{20}$. Neste sentido, acredita-se que embora muitos pacientes tenham relatado não seguirem as recomendações dietéticas, ao que parece, pelos resultados encontrados, apresentam uma preocupação com uma dieta adequada ao seu estado geral de saúde, em especial, quanto ao controle do metabolismo de cálcio e fósforo. No estudo de Pinto et al. (2009) ${ }^{15}$, a ingestão fosfórica diária média dos pacientes em hemodiálise foi de $958 \mathrm{mg} / \mathrm{dia}$, praticamente o dobro do que encontramos. Possivelmente essa diferença se deve ao fato da metodologia e instrumentos diferenciados em ambos os estudos, especialmente no que se refere ao recordatório alimentar, que no presente estudo foi de 24 h e no outro estudo de Pinto et al (2009) ${ }^{15}$ foi de três dias.

As alterações de elevação dos níveis séricos de fósforo estão entre os principais elementos a sinalizarem o início dos distúrbios do metabolismo de cálcio e fósforo e, por isso, são chamados de preditores do HPT2. Diante dessa observação, entendese que os achados até aqui descritos vão de encontro com a alta prevalência HPT2 encontrada, pois os ditos preditores desse quadro se encontravam em níveis séricos fisiológicos. Porém, não se deve subestimar a presença majoritária da HPT2 e, por isso, investigações detalhadas de outras possíveis causas desse distúrbio são necessárias, até pela possível instalação de complicações adicionais que são as doenças cardiovasculares e o consequente aumento do número de óbitos.

Sobre as recomendações alimentares específicas de proteínas para pacientes com doença renal crônica, de acordo com as diretrizes estabelecidas pelo Kidney Disease: 
Improving Global Outcomes( KDIGO 2017) ${ }^{20}$ a ingestão de proteínas deve ser de pelo menos 1,2g ptn/kg/dia para pacientes com doença renal crônica em hemodiálise, sem distinção entre os grupos com e sem HPT2. Embora os pacientes com HPT2 envolvidos nesse trabalho tenham consumido mais proteína do que aqueles sem HPT2, ainda tiveram uma ingesta proteica abaixo dos valores recomendados. Esses resultados corroboram Pinto et al.(2009) ${ }^{15}$ que em seu estudo observacional, encontraram uma ingestão proteica em pacientes dialíticos em torno de $0,9 \mathrm{~g}$ $\mathrm{ptn} / \mathrm{kg} /$ dia. Chegaram à conclusão que esse valor é suficiente para manter o paciente com estado nutricional adequado e com um balanço nitrogenado neutro. ${ }^{16}$

Resgatando agora a importância da albumina sérica como marcador de estado nutricional em pacientes em hemodiálise, destaca-se que os valores dessa proteína podem ser influenciados por diversos fatores como idade, meia vida e condições subjacentes. É correto afirmar que valores muito abaixo daqueles considerados fisiológicos, possuem forte relação com a desnutrição energético proteica e aumento de mortalidade, como aponta Santos et al. $(2004)^{16}$. Tendo em vista que a população total do trabalho atual se encontrava com valores adequados de albumina sérica, é admissível um estado nutricional energético proteico preservado dessa mesma população.

No que se refere à média de creatinina sérica, em ambos os grupos, esta se apresentou muito acima do valor de referência ( 0,6 a 1,3 $\mathrm{mg} / \mathrm{dL})$ para pessoas sem doença renal crônica. Sabe-se que a hemodiálise é capaz de equilibrar os valores séricos de creatinina, porém, dificilmente serão alcançados valores considerados fisiológicos como aqueles encontrados em pessoas hígidas ${ }^{19}$. Acredita-se que, os valores de creatinina aqui encontrados devem ser utilizados não como marcadores da efetividade da diálise, mas como indicadores complementares do estado nutricional dos pacientes. Isso é dito pois, os níveis séricos da creatinina são produto final da atividade muscular e, por vezes, após hemodiálise esses valores muito baixos ou próximos dos valores de referências para pessoas saudáveis, poderiam indicar a falta de atividade muscular representada por um estado nutricional comprometido e, não necessariamente, indicar a eficácia da hemodiálise ${ }^{21}$.

Quanto aos resultados antropométricos, a principal medida utilizada foi o índice de massa corporal (IMC) por categorias, sendo os valores encontrados similares aos de outros estudos em pacientes em hemodiálise ${ }^{22-25}$ e compatíveis com os valores encontrados no censo nacional de nutrição em pacientes em HD de $2010^{26}$ e no censo 
de diálise brasileiro de $2018^{27}$. Os resultados do presente estudo, também foram semelhantes aos do estudo nacional desenvolvido por Alvarenga et al. (2017) ${ }^{22}$ que encontraram $11,1 \%$ com baixo peso, $50 \%$ de eutróficos e 38,9\% com excesso de peso. E também semelhantes aos de um estudo internacional de Jager et al. (2001) ${ }^{23}$ que ao avaliar uma população em HD na Holanda apresentou 16\%, 50\% e 34\% referentes ao baixo peso, eutróficos e com excesso de peso, respectivamente. No que diz respeito especificamente a porcentagem de pacientes com excesso de peso os resultados são preocupantes, haja vista que a obesidade exerce influência fundamental na progressão da disfunção renal e pode ter contribuído para a falência renal desses pacientes. Além disso, a maior área corporal precisa de um reajuste de parâmetros da diálise para promover maior filtração. Esse fato, no entanto, agrega dificuldades ao procedimento de diálise e impõe maiores riscos cardiovasculares, de internação e de morte.

Ainda com relação aos resultados do IMC citados, é importante destacar que todos os estudos utilizaram a classificação de IMC padronizada pela Organização Mundial da Saúde (OMS, 2000) ${ }^{28}$ e foram encontrados resultados semelhantes ao nosso, que também utilizamos a mesma referência. Já quando Santos et al. (2013) 24 utilizaram a classificação sugerida por Riella ${ }^{29}$, que é uma classificação específica de IMC para pacientes com doenças renais, encontraram um número muito maior de desnutridos. Conseguiram encontrar diferenças significativas de classificações sendo $0 \%$ de desnutridos ao utilizar a classificação da $\mathrm{OMS}^{28}$, contra $50 \%$ de desnutridos ao utilizar a classificação proposta por Riella ${ }^{29}$. Essa constatação sugere que talvez ao utilizar outras classificações adaptadas para a população específica de pacientes com IRC, pode-se identificar mais pacientes desnutridos, uma vez que o valor de corte para o IMC de desnutrição passaria a ser menor que $25 \mathrm{~kg} / \mathrm{m}^{2}$ e não menor que $18,5 \mathrm{~kg} / \mathrm{m}^{2}$.

Por fim, sem perder de vista a importância dos dados encontrados relacionados à ingestão de fósforo, vitamina $\mathrm{D}$ e micronutrientes em geral, além do estado nutricional desses pacientes e considerando a escassez de trabalhos com esse foco, registra-se algumas limitações. A primeira a ser citada é o delineamento transversal que restringe o estabelecimento de causa - efeito entre as variáveis estudadas. Estudos longitudinais são necessários para identificação dos riscos de estado nutricional ao longo do tempo em hemodiálise com quadro simultâneo de HPT2. Além disso, cita-se a utilização do instrumento para coleta de dados sobre o 
Aryell David Proença, Carolina Lacorte Galina, Luísa Godoi Lopes, Monike Santana Gobbo, Victor Ribeiro Miamoto, Yuri Gam Faria, João Victor Marques Guedes, Márcia Christina Caetano Romano, Fernanda Cecília Dias Chula, Yoshimi Jose Ávila Watanabe, Alba Otoni I Comparação do estado nutricional em pacientes com insuficiência renal crônica com e sem hiperparatireoidismo secundário

consumo alimentar chamado "recordatório alimentar de 24 horas". Esse instrumento embora muito utilizado na prática clínica e em muitos estudos importantes sobre estado nutricional, pode apresentar fragilidade no sentido de representar o consumo pontual e não habitual dos participantes. No entanto, o recordatório de 24 horas é o mais utilizado em estudos transversais, uma vez que a aplicação do Questionário de Frequência Alimentar (QFA) é dispendiosa, demorada e, por isso, mais factível em estudos do tipo longitudinal. Salienta-se que, mesmo frente às limitações, estudos como este são de grande valia para se conhecer o perfil nutricional do paciente com IRC em hemodiálise e HPT2 e todas as implicações consequentes a essas condições de saúde.

\section{CONCLUSÃO}

Maiores consumos de micronutrientes, em especial de vitamina D e fósforo, foram encontrados entre pacientes com IRC e HPT2. Contudo, foi um consumo abaixo do recomendado, além de manutenção de níveis séricos fisiológicos desses mesmos metabólitos, indicando atendimento, por parte desses pacientes, das recomendações específicas para tratamento do HPT2.

Não houve diferença estatística no risco nutricional avaliado pelo instrumento MIS e recordatório alimentar entre os pacientes com e sem HPT2, e nem em relação ao IMC quando comparados esses mesmos grupos. No entanto, estudos longitudinais são necessários para estabelecimento de causa e efeito dos fatores determinantes do HPT2 e o real estado nutricional dessas pessoas. Além disso, esse tipo de delineamento de pesquisa permite um acompanhamento mais fidedigno do padrão alimentar e aponta a direção de uma intervenção precoce que poderá minimizar os impactos de uma dieta não ajustada à condição específica de saúde de cada paciente.

\section{REFERÊNCIAS}

1. Siviero PCL, Machado CJ, Cherchiglia ML. Insuficiência renal crônica no Brasil segundo enfoque de causas múltiplas de morte. Cad Saúde Coletiva. 2014;22(1):75-85.

2. Bastos MG, Kirsztajn GM. Artigo de revisão | review Article Correspondência para. J Bras Nefrol [Internet]. 2011;33(1):93-108. Available from: http://www.scielo.br/pdf/jbn/v33n1/v33n1a13.pdf

3. Alves LF, Abreu TT de, Neves NCS, Morais FA de, Rosiany IL, Oliveira Júnior WV de, et al. Prevalence of chronic kidney disease in a city of southeast Brazil. J Bras Nefrol [Internet]. 2017;39(2):126-34. Available from: http://www.gnresearch.org/doi/10.5935/0101-2800.20170030 
Aryell David Proença, Carolina Lacorte Galina, Luísa Godoi Lopes, Monike Santana Gobbo, Victor Ribeiro Miamoto, Yuri Gam Faria, João Victor Marques Guedes, Márcia Christina Caetano Romano, Fernanda Cecília Dias Chula, Yoshimi Jose Ávila Watanabe, Alba Otoni | Comparação do estado nutricional em pacientes com insuficiência renal crônica com e sem hiperparatireoidismo secundário

4. Ministério da Saúde (BR), Secretaria de Atenção à Saúde. Diretrizes Clínicas para o cuidado ao paciente com doença renal crônica no Sistema Único de Saúde. 2014;(1):1-37.

5. Sociedade Brasileira de Nefrologia. Censo 2014. Censo da Sociedade Brasileira de Nefrologia 2014 - Censo de Diálise. 2014. p. 1-137.

6. Bover J, Cozzolino M. Mineral and bone disorders in chronic kidney disease and end-stage renal disease patients: new insights into vitamin $D$ receptor activation. Kidney Int Suppl [Internet]. 2011;1(4):122-9. Available from: http://www.ncbi.nlm.nih.gov/pubmed/25018911

7. Abrita RR, Pereira B dos S, Fernandes N da S, Abrita R, Huaira RMNH, Bastos MG, et al. Evaluation of prevalence, biochemical profile, and drugs associated with chronic kidney disease-mineral and bone disorder in 11 dialysis centers. Brazilian J Nephrol. 2018;40(1):26-34.

8. Hogan Jonathan GS. Regulation of calcium and phosphate balance - UpToDate [Internet]. 2016 [cited 2016 May 2]. Available from:

https://www.uptodate.com/contents/regulation-of-calcium-and-phosphatebalance

9. Quarles LD, Berkoben M. Bone biopsy and the diagnosis of renal osteodystrophy - UpToDate [Internet]. 2016 [cited 2016 May 7]. Available from:

https://www.uptodate.com/contents/bone-biopsy-and-the-diagnosis-ofrenal-osteodystrophy

10. Custódio MR, Canziani MEF, Moysés RMA, Barreto FC, Neves CL, de Oliveira RB, et al. Protocolo clínico e diretrizes terapêuticas para o tratamento do hiperparatireoidismo secundário em pacientes com doença renal crônica. J Bras Nefrol 'orgão Of Soc Bras e Latino-Americana Nefrol. 2013;35(4):308-22.

11. Kalantar-Zadeh K, Kopple JD, Block G, Humphreys MH. A malnutritioninflammation score is correlated with morbidity and mortality in maintenance hemodialysis patients. Am J Kidney Dis. 2001;38(6):1251-63.

12. Fetter RL, Bigogno FG, Oliveira FGP de, Avesani CM. Cross-cultural adaptation to Portuguese of tools for assessing the nutritional status of patients on dialysis. J Bras Nefrol [Internet]. 2014;36(2):176-85. Available from: http://www.gnresearch.org/doi/10.5935/0101-2800.20140028

13. Yamada K, Furuya R, Takita T, Maruyama Y, Yamaguchi Y, Ohkawa S, et al. Simplified nutritional screening tools for patients on maintenance hemodialysis. Am J Clin Nutr. 2008;87(1):106-13.

14. Rezende LTT, Cuppari L, Carvalho AB, Canziani MEF, Manfredi SR, Cendoroglo $M$, et al. Nutritional status of hemodialysis patients with secondary hyperparathyroidism. Brazilian J Med Biol Res. 2000;33(11):1305-11.

15. Pinto DE, Ullmann LS, Burmeister MM, Antonello ICF, Pizzato A. Associações entre ingestão energética, proteica e de fósforo em pacientes portadores de doença renal crônica em tratamento hemodialítico. J Bras Nefrol [Internet]. 2009;31:269-76. Available from:

http://www.scielo.br/scielo.php?script=sci_arttext\&pid=S010128002009000400005\&lng=pt\&nrm=iso\&tlng=pt

16. Santos NSJ, Draibe SA, Kamimura MA, Cuppari L. Serum albumin as nutritional marker of hemodialysis patients. Rev Nutr. 2004;17(3):339-49. 
Aryell David Proença, Carolina Lacorte Galina, Luísa Godoi Lopes, Monike Santana Gobbo, Victor Ribeiro Miamoto, Yuri Gam Faria, João Victor Marques Guedes, Márcia Christina Caetano Romano, Fernanda Cecília Dias Chula, Yoshimi Jose Ávila Watanabe, Alba Otoni | Comparação do estado nutricional em pacientes com insuficiência renal crônica com e sem hiperparatireoidismo secundário

17. Campos SR, Gusmão MHL, Almeida AF, Pereira LJC, Sampaio LR, Medeiros JMB. Estado nutricional e ingestão alimentar de pacientes em diálise peritoneal contínua com e sem hiperparatireoidismo secundário. J Bras Nefrol [Internet]. 2012 Jun;34(2):170-7. Available from:

http://www.scielo.br/scielo.php?script=sci_arttext\&pid=S010128002012000200010\&lng=pt\&nrm=iso\&tlng=en

18. Gueiros JEB, Hernandes FR, Karohl C, Jorgetti V. Prevenção e tratamento do hiperparatireoidismo secundário na DRC. J Bras Nefrol [Internet]. 2011 Apr;33(Tabela 1):7-14. Available from: http://www.scielo.br/scielo.php?script=sci_arttext\&pid=S010128002011000500003\&lng=pt\&nrm=iso\&tlng=en

19. Draczevski L, Teixeira ML. Avaliação do Perfil Bioquímico e Parâmetros Hematológicos em Pacientes Submetidos à Hemodiálise. Rev Saúde e Pesqui. 2011;4:15-22.

20. Isakova T, Nickolas TL, Denburg M, Yarlagadda S, Weiner DE, Gutiérrez OM, et al. KDOQI US Commentary on the 2017 KDIGO Clinical Practice Guideline Update for the Diagnosis, Evaluation, Prevention, and Treatment of Chronic Kidney Disease-Mineral and Bone Disorder (CKD-MBD). Am J Kidney Dis. 2017;70(6):737-51.

21. Ferraz SF, Freitas ATV de S, Vaz IMF, Campos MIVAM, Peixoto M do RG, Pereira ERS. Nutritional status and interdialytic weight gain of chronic hemodialysis patients. J Bras Nefrol [Internet]. 2015;37(3):306-14. Available from: http://www.gnresearch.org/doi/10.5935/0101-2800.20150050

22. Alvarenga L de A, Andrade BD, Moreira MA, Nascimento R de P, Macedo ID, Aguiar AS de. Nutritional profle of hemodialysis patients concerning treatment time. J Bras Nefrol [Internet]. 2017;39(3):283-6. Available from: http://www.gnresearch.org/doi/10.5935/0101-2800.20170052

23. Jager KJ, Merkus MP, Huisman RM, Boeschoten EW, Dekker FW, Korevaar JC, et al. Nutritional status over time in hemodialysis and peritoneal dialysis. J Am Soc Nephrol [Internet]. 2001;12(6):1272-9. Available from: http://www.ncbi.nlm.nih.gov/pubmed/11373352

24. Santos ACB dos, Machado M do C, Pereira LR, Abreu JLP, Lyra MB. Association between the level of quality of life and nutritional status in patients undergoing chronic renal hemodialysis. J Bras Nefrol [Internet]. 2013;35(4):279-88. Available from: http://www.gnresearch.org/doi/10.5935/0101-2800.20130047

25. Martone AP, Coutinho V, Liberali R. Avaliação do estado nutricional de pacientes renais crônicos em hemodiálise do Instituto de Hipertensão Arterial e Doenças Renais de Campo Grande-MS. Rev Bras Nutr Clin. 2012;27(1):9-16.

26. Biavo BMM, Martins CTB, Cunha LM, Araujo ML de, Ribeiro MMC, Sachs A, et al. Nutritional and epidemiological aspects of patients with chronic renal failure undergoing hemodialysis from Brazil, 2010. J Bras Nefrol [Internet]. 2012;34(3):206-15. Available from: http://www.gnresearch.org/doi/10.5935/0101-2800.20120001

27. Nefrologia SB de. Censo da Sociedade Brasileira de Nefrologia 2018 - Censo de Diálise. 2018. p. 1-44. 
Aryell David Proença, Carolina Lacorte Galina, Luísa Godoi Lopes, Monike Santana Gobbo, Victor Ribeiro Miamoto, Yuri Gam Faria, João Victor Marques Guedes, Márcia Christina Caetano Romano, Fernanda Cecília Dias Chula, Yoshimi Jose Ávila Watanabe, Alba Otoni | Comparação do estado nutricional em pacientes com insuficiência renal crônica com e sem hiperparatireoidismo secundário

28. World Health Organization. Obesity:preventing and managing the global epidemic. World Heal Organ - Tech Rep Ser. 2000;1-268.

29. Riella Miguel C MC. Nutrição e o rim. In: Nutrição e o rim. 2.ed. Rio de Janeiro: Guanabara Koogan; 2013. p. p114-31

Submissão: 03/12/2019

Aprovação: $12 / 11 / 2020$ 